\title{
A Gallery of Iterated Correspondences
}

\author{
Shaun Bullett and Christopher Penrose
}

\section{CONTENTS}

1. Introduction

2. Quadratic Correspondences and Their Graphs

3. Resolvable Correspondences

4. Directionalities, Limit Sets and Fundamental Domains

5. Combining Quadratic-Like Julia Sets

6. Mating Quadratic Maps with the Modular Group

7. Perturbing Circle-Packing Kleinian Groups as Correspondences

8. More Exotic Examples: Tweaked Julia Sets and Bull's-Eyes 9. Concluding Remarks

Acknowledgements

Bibliography
This research was supported by SERC. Bullett also thanks IHES for its warm hospitality during the course of the work presented here.
Algebraic correspondences on the Riemann sphere generalise both Kleinian groups and rational maps. We classify quadratic correspondences satisfying certain "diagram conditions" and derive canonical forms for them. We exhibit examples for which the regular set and limit set are both nontrivial, and show that such examples can simultaneously exhibit Kleinian-grouplike and rational-map-like behaviour. We also state some general theorems and conjectures.

\section{INTRODUCTION}

A nondegenerate algebraic correspondence of bidegree $(m, n)$ on a closed Riemann surface $\mathcal{S}$ is an algebraic hypersurface $f \subset \mathcal{S} \times \mathcal{S}$ such that the projections of $f$ onto the two copies of $\mathcal{S}$ are locally injective almost everywhere and of degrees $m$ and $n$ respectively [Semple and Roth 1949]. If $f$ and $f^{\prime}$ are correspondences on the same surface $\mathcal{S}$, their composite $f^{\prime \prime}$ is the set of pairs $(z, w)$ such that there exists $v \in \mathcal{S}$ with $(z, v) \in f$ and $(v, w) \in f^{\prime}$.

When $\mathcal{S}$ is the Riemann sphere $\hat{\mathbb{C}}$, the hypersurface $f$ is defined by a polynomial equation

$$
p(z, w)=0
$$

and we think of $f$ as the graph of the multivalued function $z \mapsto w$ (which we also denote by $f$ ) defined implicitly by this equation. Composition of correspondences is now just the usual composition of (multivalued) functions.

Here are some examples of correspondences on the Riemann sphere:

Example 1.1. (a) Quadratic maps $z \mapsto z^{2}+c$ are $(2,1)$ correspondences, since they can be written in the form $w-\left(z^{2}+c\right)=0$. 
(b) The (two-valued) inverse of a quadratic map is a $(1,2)$ correspondence, since it can be written $z-\left(w^{2}+c\right)=0$.

(c) The generators $z \mapsto z+1$ and $z \mapsto-1 / z$ of the modular group $\operatorname{PSL}(2, \mathbb{Z})$ define a $(2,2)$ correspondence $(w-(z+1))(w+1 / z)=0$.

Note that in (c) the grand orbit of any point $z$ under the correspondence (allowing arbitrary forward and backward iterations) is precisely the orbit of $z$ under the modular group. More generally, to any set of $n$ elements of $\operatorname{PSL}(2, \mathbb{C}$ ) (acting by fractional linear transformations $z \mapsto(a z+b) /(c z+d))$ we can associate an $(n, n)$ correspondence on the Riemann sphere, and the grand orbits will be those of the group generated by the transformations.

It is well known that in example (b) the forward orbit of a generic point accumulates on a set that is independent of the generic point chosen (the $\mathrm{Ju}$ lia set of the quadratic map of example (a)), and that the same applies for any orbit of a discrete subgroup of PSL $(2, \mathbb{C}$ ) (in the case of the modular group, this limit set is the real line union infinity).

Discrete subgroups of $\operatorname{PSL}(2, \mathbb{C})$ are of two types [Maskit 1987]: Kleinian groups of the first kind, which have limit set the whole sphere, and those of the second kind, where the complement of the limit set (the regular set) is made up of copies of a fundamental domain.

For rational maps [Beardon 1991; Douady and Hubbard 1982; 1984] there is an analogous dichotomy: the Julia set may be either the whole sphere or a proper subset. The action of a rational map on the complement of its Julia set (the Fatou set) again has a kind of "regular" behaviour, and there is even the analogue of a "fundamental domain" in the hyperbolic case, that is, when the Fatou set is made up of basins of attraction of periodic orbits [Sullivan 1984].

Münzner and Rasch [Rasch 1988; Münzner and Rasch 1991] have shown that the same dynamical dichotomy exists on the space of orbits of an algebraic correspondence under one-way iteration, and that much of the classical Fatou-Julia theory extends to this situation. Our interest here is quite different. We are concerned with the dynamical dichotomy on the space on which the correspondence acts (the Riemann sphere), and with grand orbits of correspondences (mixed forward and backward iteration), rather than one-way iteration.

For generic algebraic correspondences on the Riemann sphere, one might expect generic grand orbits to be dense. This is indeed the case for $(2,2)$ correspondences, as is not too difficult to prove. However, there are also large classes of $(2,2)$ correspondences that have a "global limit set" that is a proper subset of the sphere, and where there is a "fundamental domain" for the action on the complement of this limit set, just as for Kleinian groups of the second kind and hyperbolic rational maps. Examples vary from behaviour very like that of a Kleinian group to very like that of a rational map, with some intriguing cases in between.

In this paper we summarise our general results concerning iterated $(2,2)$ correspondences on the Riemann sphere, survey the examples we have constructed so far - in particular, "matings" between Kleinian group and rational map actions - and indicate some directions for further study.

Our motivation for undertaking this investigation was the striking series of results of Sullivan obtained by applying quasiconformal deformation theory to both rational maps and Kleinian groups [Sullivan 1984; 1985a; 1985b]. Our hope was that by studying iterated correspondences we could obtain further insight into how these classes of dynamical systems are related. The results outlined in this paper are a step in that direction: we believe the examples also have considerable interest in their own right.

\section{QUADRATIC CORRESPONDENCES AND THEIR GRAPHS}

We restrict attention to quadratic correspondences, that is, nondegenerate $(2,2)$ correspondences on the Riemann sphere. Much of what follows can be generalised to higher degrees and to other Riemann 
surfaces, but there are often considerable complications.

A quadratic correspondence is an algebraic hypersurface $f \subset \hat{\mathbb{C}} \times \hat{\mathbb{C}}$, defined by an equation $p(z, w)=0$, where $p$ is of degree two in each of $z$ and $w$ separately. Nondegeneracy means that every nonconstant factor of $p(z, w)$ involves both $z$ and $w$. An algebraic hypersurface has a normalisation or desingularisation $Q: \Gamma \rightarrow f$, where $\Gamma=$ $\Gamma(f)$ is a closed Riemann surface and $Q_{-}=\pi_{-} Q$ and $Q_{+}=\pi_{+} Q$ are holomorphic maps of degree two from $\Gamma$ to $\hat{\mathbb{C}}$. Here $\pi_{-}$and $\pi_{+}$denote the two projections from $\hat{\mathbb{C}} \times \hat{\mathbb{C}}$ onto $\hat{\mathbb{C}}$. See [Semple and Roth 1949; Shafarevich 1974] for details.

Formally, to regard $p(z, w)=0$ as a subset of $\hat{\mathbb{C}} \times \hat{\mathbb{C}}=\mathbb{C P}^{1} \times \mathbb{C P}^{1}$ we should homogenise the polynomial $p(z, w)$ into a homogeneous poynomial $P\left(\left[z, t_{1}\right],\left[w, t_{2}\right]\right)$ of degree four, where each term is separately of total degree two in $z$ and $t_{1}$, and of total degree two in $w$ and $t_{2}$. However we shall follow the usual convention and suppress the homogenising variables, at the expense of allowing $\infty$ as a possible value for $z$ and $w$.

The correspondence defined by $p(z, w)=0$ lifts to a correspondence on the graph $f$ itself, defined by the subset $F \subset f \times f$ consisting of pairs of pairs $((z, w),(w, \tau)) \in f \times f$, that is, satisfying $p(z, w)=0$ and $p(w, \tau)=0$. Normalising $f$ via $Q: \Gamma \rightarrow f$, we define $\tilde{f} \subset \Gamma \times \Gamma$ to consist of pairs $(s, t)$ such that $Q_{+}(s)=Q_{-}(t)$, where, as before, $Q_{-}=\pi_{-} Q$ and $Q_{+}=\pi_{+} Q$. Note that $\tilde{f}$ is an algebraic hypersurface in $\Gamma \times \Gamma$. We call $\tilde{f}$ the lift of the correspondence $f$.

Observe that we may write $Q_{+} Q_{-}^{-1}$ for $f$ and $Q_{-}^{-1} Q_{+}$for $\tilde{f}$.

Remark. Given any Riemann surface $\Gamma$ and any pair of degree-two holomorphic maps $Q_{-}, Q_{+}: \Gamma \rightarrow \hat{\mathbb{C}}$, the composition $Q_{+} Q_{-}^{-1}$ defines a correspondence. When $\Gamma$ has genus two or higher, the covering involutions of $Q_{+}$and $Q_{-}$are identical, since $\Gamma$ carries a unique hyperelliptic involution, and thus $Q_{+}=$ $M Q_{-}$for some Möbius transformation $M$ of $\hat{\mathbb{C}}$. It follows that $Q_{+} Q_{-}^{-1}(=M)$ is $(1,1)$. When $\Gamma$ has genus zero or 1, it is a pair of spheres (each projecting one-to-one onto $\hat{\mathbb{C}}$ ), or a single sphere (branch-covering $\hat{\mathbb{C}}$ ), or a torus (an elliptic curve branch-covering $\hat{\mathbb{C}}$ ). In each of these three cases one can apply standard theory of maps $\Gamma \rightarrow \widehat{\mathbb{C}}$ to show that $Q_{+} Q_{-}^{-1}$ is algebraic and of bidegree either $(1,1)$ or $(2,2)$.

\section{Singular Points and the Topological Type of the Graph}

Singular points for an algebraic correspondence $f$ on any closed Riemann surface are of three types. Let $Q: \Gamma \rightarrow f$ be the normalisation of $f$ and let $\pi_{-}, \pi_{+}$be the two projections of $f$. As before write $Q_{-}$for $\pi_{-} Q$ and $Q_{+}$for $\pi_{+} Q$. The types of special point on $f$ are best described after lifting to $\Gamma$. They are

- the critical points of $Q_{-}$;

- the critical points of $Q_{+}$; and

- the singular points of $Q$.

As we shall see, the possibilities for singular points of $Q$, and for points which are simultaneously of different types, are quite limited for $(2,2)$ correspondences.

The special points of various types project onto singular points of the correspondence on the dynamical plane, the Riemann sphere. By a forward singular point of the correspondence $f=Q_{+} Q_{-}^{-1}$ we mean a point $z$ that has fewer images $w$ under $f$ than its immediate neighbours, and by a backward singular point we mean a point $w$ that has fewer preimages than its immediate neighbours. Thus forward singular points are critical values of $Q_{-}$or else $Q_{-}$-images of singular points of $Q$, and backward singular points are critical values of $Q_{+}$or else $Q_{+}$-images of singular points of $Q$.

A quadratic correspondence on the sphere has four forward singular points and four backward ones, when counted with multiplicity [Semple and Roth 1949, Ch. IV]. If we write the correspondence in the form

$$
w^{2} A(z)+w B(z)+C(z)=0
$$


where $A, B$ and $C$ are quadratic polynomials, the forward singular points are given by the solutions of

$$
B(z)^{2}=4 A(z) C(z)
$$

Let $z=z_{0}$ be a solution of (2.2) of multiplicity $r$ and let $w=w_{0}$ be the (unique) corresponding value of $w$. If we choose a coordinate system in which $w_{0} \neq \infty$, the solution of (2.1) has the local form

$$
w-w_{0}=\left(z-z_{0}\right) \varphi_{1}(z) \pm\left(z-z_{0}\right)^{r / 2} \varphi_{2}(z),
$$

where $\varphi_{1}$ and $\varphi_{2}$ are analytic and $\varphi_{2}\left(z_{0}\right) \neq 0$.

If $r$ is even, $\left(z_{0}, w_{0}\right)$ is a double point of the correspondence, a point where the two sheets cut transversely (if $r=2$ ) or touch (if $r=4$ ). If $r$ is odd, when we desingularise $f$ there is a coordinate $Z$ on $\Gamma$ having $Z^{2}=z-z_{0}$. Thus when lifted to $\Gamma$ the point $\left(z_{0}, w_{0}\right)$ becomes a critical point of $Q_{-}$. For $r=1$, Equation (2.3) gives

$$
w-w_{0}=\lambda Z+\text { higher terms in } Z,
$$

with $\lambda \neq 0$, so $\left(z_{0}, w_{0}\right)$ is an ordinary point of $Q_{+}$, while for $r=3$ the equation makes $w-w_{0}$ equal to a sum of terms of degree two or higher in $Z$, so $\left(z_{0}, w_{0}\right)$ is a critical point of $Q_{+}$as well as of $Q_{-}$.

To sum up:

- for $r=1, z$ is a critical point of $Q_{-}$;

- for $r=2, z$ is a double point of $Q$;

- for $r=3, z$ is a critical point of $Q_{-}$and of $Q_{+}$, and a nonsmooth point of $Q$;

- for $r=4$, two double points of $Q$ coincide at $z$.

We obtain different topological types for the normalisation $\Gamma$ of the graph of a quadratic correspondence $f$, depending on the various possibilities for the multiplicities of the roots of (2.2). These types are computed by calculating the Euler characteristic of $\Gamma$, using the branched double covering map $Q_{-}: \Gamma \rightarrow \hat{\mathbb{C}}$.

(a) If there are four distinct roots, $\Gamma$ is a torus ( $f$ is an elliptic curve). (b) If there are three roots, one of which is double, or two roots, one of which is triple, $\Gamma$ is a sphere, self-intersecting or with a nonsmooth point.

(c) If there are two double roots or one quadruple root, $f$ is reducible, a pair of spheres intersecting at two points or touching at one. Its normalisation $\Gamma$ is a disjoint pair of spheres.

A quadratic correspondence is equivalent to a subgroup of $\operatorname{PSL}(2, \mathbb{C})$ (with two generators) if and only if its graph $f$ is of type (c) above.

As an example of an explicit computation of singular points consider the arithmetic-geometric mean correspondence

$$
4 z w^{2}=(z+1)^{2},
$$

so-called because it sends $z$ to the ratio $w$ between the arithmetic and geometric means of 1 and $z$.

In the notation of (2.1), we have

$$
A(z)=4 z, B(z)=0, C(z)=-(z+1)^{2} .
$$

Thus (2.2) becomes

$$
16 z(z+1)^{2}=0,
$$

with solutions $z=-1$ of multiplicity two and $z=$ $0, \infty$ of multiplicity one. Therefore $Q_{-}$has two critical values $z=0$ and $z=\infty$, and $Q: \Gamma \rightarrow f$ has one double point $(z, w)=(-1,0)$. It follows that $\Gamma$ is a single sphere.

\section{Maps of Pairs and Maps of Triples}

A quadratic correspondence $f$ comes equipped with two involutions $I_{-}, I_{+}$, the covering involutions for the two projections $\pi_{-}, \pi_{+}$of the graph $f$ onto $\hat{\mathbb{C}}$. We shall use the same notation $I_{-}, I_{+}$for the lifts of these involutions to the normalisation $\Gamma$ of $f$, namely the covering involutions for $Q_{-}=\pi_{-} Q$ and $Q_{+}=\pi_{+} Q$.

Remark. For correspondences of higher degree, the analogues of $I_{-}$and $I_{+}$are themselves correspondences rather than group elements.

Commutation conditions on $I_{-}$and $I_{+}$translate into diagram conditions on the two-valued map 
$z \mapsto w$ defined by the equation $p(z, w)=0$ of $f$. The diagram conditions that will concern us are those defining maps of pairs and maps of triples.

We say that a quadratic correspondence is a map of pairs if $z_{1} \mapsto w_{1}, z_{1} \mapsto w_{2}$ and $z_{2} \mapsto w_{1}$ imply $z_{2} \mapsto w_{2}$. Diagramatically,

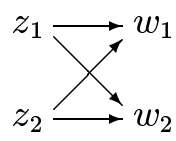

Since $I_{-}$is the involution on $f$ that interchanges $\left(z_{1}, w_{1}\right)$ and $\left(z_{1}, w_{2}\right)$, and $I_{+}$interchanges $\left(z_{1}, w_{1}\right)$ and $\left(z_{2}, w_{1}\right)$, it is an elementary observation that $f$ is (the graph of) a map of pairs if and only if $I_{-} I_{+}=I_{+} I_{-}$.

We say that a quadratic correspondence is a map of triples if, for every $z_{1}$, there are points $z_{2}, z_{3}, w_{1}$, $w_{2}$ and $w_{3}$, not necessarily distinct, such that the images of the $z_{i}$ and the preimages of the $w_{i}$ form a diagram

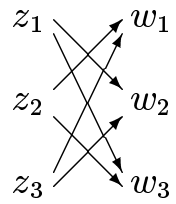

We say that a map of triples $f$ is reversible if the map $z_{i} \mapsto w_{i}$ that exists as a consequence of (2.6) is an involution of $\hat{\mathbb{C}}$. It is an elementary observation that $f$ is (the graph of) a map of triples if and only if $I_{-} I_{+} I_{-}=I_{+} I_{-} I_{+}$, and that the map of triples is reversible if and only if $I_{-} I_{+} I_{-}(z, w)=(J w, J z)$ for an involution $J$.

Of course one can consider other commutation conditions - indeed we do so in [Bullett and Penrose a] - but the conditions just given are particularly appealing, in that correspondences $f$ satisfying them have straightforward canonical forms for their equations $p(z, w)=0$, as we now show.

We say that a correspondence $f$ is separable if it has an equation of the form $h_{1}(z)=h_{2}(w)$, where $h_{1}$ and $h_{2}$ are rational functions.

Lemma 2.1. A quadratic correspondence $f$ is a map of pairs if and only if it is separable.
Proof. Let $f$ have equation $h_{1}(z)=h_{2}(w)$. Given any $z_{1} \in \hat{\mathbb{C}}$, let $\zeta=h_{1}\left(z_{1}\right)$. Then $z_{1}$, together with the other root $z_{2}$ of $h_{1}(z)=\zeta$ and the two roots $w_{1}$ and $w_{2}$ of $h_{2}(w)=\zeta$, obey diagram (2.5), so $f$ is a map of pairs. Conversely, if $f$ is a map of pairs, then it is immediate from (2.5) that $f$ has an equation of the form $j_{1}(z)=M j_{2}(w)$, where $j_{1}$ identifies $z_{1}$ with $z_{2}, j_{2}$ identifies $w_{1}$ with $w_{2}$, and $M$ is a Möbius transformation.

Remark. If $f$ is a map of pairs, it has the form $z \mapsto$ $h_{2}^{-1} h_{1}(z)$, and hence it is the lift of the "pushed down" correspondence $z \mapsto h_{1} h_{2}^{-1}(z)$ to its graph. See the discussion of lifts of correspondences earlier in this section.

Lemma 2.2. A quadratic correspondence $f$ is a map of triples if and only if there exists a rational map $C$ of degree three and a Möbius transformation $M$ such that $f$ has equation $(C(w)-C(M z)) /(w-$ $M z)=0$. In addition, $f$ is a reversible map of triples if and only if $M$ is an involution.

Proof. If $f$ has equation of this form, it is easily seen to satisfy the diagram condition (2.6): just set $w_{i}=M z_{i}$ for $i=1,2,3$. Conversely, if $f$ satisfies the diagram condition, we may define $M$ to be the map that associates each $w_{i}$ to the corresponding $z_{i}$, and $C$ to be any rational function of degree three that maps each triple $\left\{z_{1}, z_{2}, z_{3}\right\}$ to a single point.

In Section 3 one of our concerns will be maps of triples where $M$ is the identity (cyclically symmetric correspondences), and in Section 6 we shall see how certain classes of reversible maps of triples can be viewed as "matings" between the modular group and quadratic maps.

\section{RESOLVABLE CORRESPONDENCES}

In this section we describe classes of correspondences which can be "resolved" to group actions by lifting to suitable covering spaces. The problem of describing their dynamics is thereby reduced to 
group theory. We first introduce one more piece of terminology.

A forward critical point of the correspondence $f=Q_{+} Q_{-}^{-1}$ is the image $Q_{-}(c) \in \hat{\mathbb{C}}$ of a critical point $c \in \Gamma$ of $Q_{+}$. The corresponding forward critical value of $f$ is $Q_{+}(c) \in \hat{\mathbb{C}}$, in other words a critical value of $Q_{+}$(and hence, as we observed in the previous section, a backward singular point of $f$ ). This agrees with the standard terminology when $f=Q_{+} Q_{-}^{-1}$ is a rational map, that is, when $Q_{-}$is a Möbius transformation.

Similarly, a backward critical point of $f$ is the image $Q_{+}\left(c^{\prime}\right) \in \hat{\mathbb{C}}$ of a critical point $c^{\prime}$ of $Q_{-}$, and $Q_{-}\left(c^{\prime}\right)$ is the corresponding backward critical value of $f$ (a forward singular point).

\section{Critically Finite Correspondences}

As for iterated rational maps, it is the behaviour of critical points that determines the overall behaviour of a correspondence under iteration. The simplest situation to analyse is when the grand orbits of all critical points are finite [Bullett 1992]. These critically finite correspondences are analogous in some ways to postcritically finite rational maps, which play an important role in that theory. However, critical finiteness in this sense is a much stronger condition than postcritical finiteness for rational maps: for example, the only critically finite rational maps of degree two are $z \mapsto z^{2}$ and $z \mapsto 1 / z^{2}$.

Any critically finite correspondence can be resolved by removing the critical orbits and then lifting to the universal cover of the resulting punctured sphere. On this universal cover, which is the complex upper half-plane if the critical orbits contain at least three points, the lifted correspondence has no critical points, forwards or backwards, and therefore factorises into biholomorphic transformations of the upper half-plane - that is to say, elements of $\operatorname{PSL}(2, \mathbb{R})$. For example, consider the arithmetic-geometric mean correspondence (2.4). This has forward critical values 1 and -1 , and backward critical values 0 and $\infty$. All these points fit together in the (finite) grand orbit

$$
\circlearrowright 1 \rightarrow-1 \rightarrow 0 \rightarrow \infty \text { ๖ }
$$

The universal cover of the sphere punctured at these four points is the complex upper half-plane $\mathbb{H}$, with covering transformation group

$$
G=\Gamma_{2}(4)=\left(\begin{array}{ll}
1(4) & 0(2) \\
0(4) & 1(4)
\end{array}\right) \subset \operatorname{PSL}(2, \mathbb{Z}) .
$$

The arithmetic-geometric mean correspondence lifts to the map $\tau \mapsto 2 \tau$ on $\mathbb{H}$, and of course also to lifts to the same map pre- or post-composed with any covering transformation. From analysis of how $\tau \mapsto 2 \tau$ interacts with the covering group it follows that all grand orbits other than the critical one are dense on the sphere. There is much interesting geometry underlying the arithmetic-geometric mean correspondence [Bullett 1991], in particular that investigated by Gauss in his remarkable work on elliptic integrals and theta functions.

Other critically finite correspondences can be resolved in a similar way. In [Bullett 1992] there is a classification of all strongly critically finite quadratic correspondences, together with the covering groups of the associated punctured spheres, and lifts of the correspondences to the upper half-plane. A correspondence is called strongly critically finite if not only are the critical orbits finite, but all points on them are critical values, either forwards or backwards. This is a technical condition that makes the classification problem easier: there are just eleven strongly critically finite quadratic correspondences, and their covering groups are all subgroups of $\operatorname{PSL}(2, \mathbb{Z})$ associated to regular solids. In each of these examples, every grand orbit of the correspondence, other than critical orbits, is dense on the Riemann sphere.

\section{Critically Resolvable Correspondences}

We say that a quadratic correspondence is critically resolvable if every critical value of $Q_{-}$is also a critical value of $Q_{+}$, and vice versa. On the dynamical plane $\hat{\mathbb{C}}$ this is the condition that every forward critical value of $f=Q_{+} Q_{-}^{-1}$ is also a backward one, and vice versa. 
Example 3.1. Consider the correspondence

$$
\left(\frac{z-1}{z+1}\right)^{2}=-4 \frac{a w}{(w-a)^{2}}
$$

This has critical points 1 and $-a$, and double points -1 and $a$, with orbits

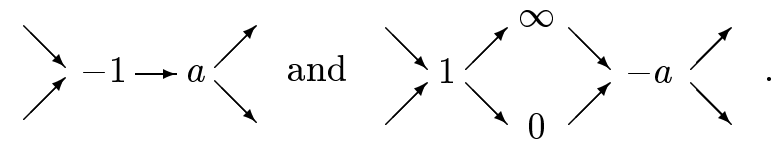

Thus 0 and $\infty$ are both forward and backward critical values. Note that the cases $a=1$ and $a=-1$ give critically finite correspondences. Indeed in these cases all orbits are finite.

Lemma 3.2. A quadratic correspondence is critically resolvable if and only if it can be written

$$
f=Q_{-} T Q_{-}^{-1}
$$

for some automorphism $T$ of $\Gamma$.

Proof. Critical values determine a degree-two map $\Gamma \rightarrow \hat{\mathbb{C}}$ up to premultiplication by an automorphism $T$ of $\Gamma$.

Remark. Since, given any $Q_{-}$and $Q_{+}$, there exist automorphisms $S$ of $\hat{\mathbb{C}}$ and $T$ of $\Gamma$ such that $Q_{+}=S Q_{-} T$, every quadratic correspondence is the composition of a critically resolvable correspondence followed by a Möbius transformation.

It follows from Lemma 3.2 that the lift $\tilde{f}$ of a critically resolvable correspondence $f$ to the normalisation $\Gamma$ of its graph is the pair of $(1,1)$ maps $\left\{T, I_{-} T\right\}$. Thus $\tilde{f}$ is a reducible correspondence. We remark that when $\Gamma$ is a pair of spheres we can distinguish between $T$ and $I_{-} T$ by the fact that one (say $T$ ) maps each sphere to itself, and the other $\left(I_{-} T\right)$ exchanges the spheres. When $\Gamma$ is a generic torus (generic means the only rotational symmetries of $\Gamma$ are of order two) we can similarly distinguish the translational lift (which we denote $T$ ) from the elliptic one $\left(I_{-} T\right)$, but when $\Gamma$ is a single sphere we have no such distinction of types of automorphism.

The following theorem is now self-evident.
Theorem 3.3. Any critically resolvable $(2,2)$ correspondence lifts to an action on $\Gamma$ of the free product $C_{\infty} * C_{2}$ of an infinite cyclic group (generated by $T$ ) with a cyclic group of order two (generated by $I_{-}$). Conversely, any action of $C_{\infty} * C_{2}$ on a Riemann surface $\Gamma$ that is topologically a pairs of spheres, a single sphere or a torus is the lift of a critically resolvable quadratic correspondence on $\hat{\mathbb{C}}$, provided that the generators $T$ and $I_{-}$of $C_{\infty} * C_{2}$ satisfy these conditions:

(i) $T$ and $I_{-}$do not commute;

(ii) if $\Gamma$ is a pair of spheres, $I_{-}$exchanges spheres and $T$ preserves them; and

(iii) if $\Gamma$ is a torus, $I_{-}$is elliptic.

Condition (i) is necessary to ensure that the correspondence is not $(1,1)$. Note also that in the case that $\Gamma$ is a pair of spheres or a generic torus Theorem 3.3 can be sharpened to state that there is a bijection between conformal conjugacy classes of critically resolvable correspondences and conformal conjugacy classes of (nonabelian) actions of $C_{\infty} * C_{2}$.

As an example of how to resolve a critically resolvable correspondence in practice, consider (3.1), which is in fact the family of all critically resolvable maps of pairs with graph a single sphere. The critical values are 0 and $\infty$, so we can resolve by substituting $Z^{2}$ for $z$ and $W^{2}$ for $w$ in the equation. We deduce that the lift of the correspondence is the group generated by the Möbius transformations

$$
T: Z \mapsto i \sqrt{a} \frac{Z+1}{Z-1} \quad \text { and } \quad I_{-} T=T I_{+},
$$

where $Z=(z, w)$ is a coordinate on $f$, and $I_{-}$: $Z \mapsto-Z$ is the covering involution for the projection $Z \mapsto z=Z^{2}$. (In this parametrization $I_{+}$is the involution $Z \mapsto 1 / Z$.)

The class of critically resolvable quadratic correspondences contains two subclasses of particular interest: symmetric correspondences, for which $z \mapsto w$ if and only if $w \mapsto z$; and cyclically symmetric correspondences [Semple and Roth 1949, Chapter IV], for which generic grand orbits have three 
points, and the correspondence maps each $z$ to the other two points on its grand orbit (equivalently, there exists a rational function $C: \hat{\mathbb{C}} \rightarrow \hat{\mathbb{C}}$ of degree three such that the correspondence is defined by $(C(w)-C(z)) /(w-z)=0)$.

It is evident that cyclically symmetric, symmetric and critically resolvable are successively weaker properties.

Lemma 3.4. A critically resolvable quadratic correspondence $f$ is symmetric if and only if one of its lifts $T$ and $I_{-} T$ to $\Gamma$ is an involution; it is cyclically symmetric if and only if one lift is an involution and the other has order three.

Proof. If either $T$ or $I_{-} T$ is an involution, it is immediate that $f$ is symmetric. For the converse, observe that if $f$ is symmetric the involution $(z, w) \mapsto$ $(w, z)$, restricted to $(z, w) \in f$, is a lift of $f$ to its graph, and hence either $T$ or $I_{-} T$ is this involution. The proof of the statement for cyclically symmetric correspondences is analogous.

We have the following immediate corollary of Theorem 3.3 and Lemma 3.4.

Corollary 3.5. Any symmetric $(2,2)$ correspondence lifts to an action of $C_{2} * C_{2}$ on $\Gamma$. Conversely, any action of $C_{2} * C_{2}$ on a Riemann surface $\Gamma$ that is topologically a pair of spheres, a single sphere or a torus is the lift of a symmetric quadratic correspondence on $\hat{\mathbb{C}}$, provided that the generators $T$ and $I_{-}$ of $C_{2} * C_{2}$ satisfy conditions (i)-(iii) of Theorem 3.3.

Every cyclically symmetric $(2,2)$ correspondence lifts to an action of the dihedral group $D_{6}$ of order six on $\Gamma$. Conversely, any action of $D_{6}$ on a Riemann surface $\Gamma$ that is topologically a pair of spheres, a single sphere or a torus is the lift of a cyclically symmetric quadratic correspondence on $\hat{\mathbb{C}}$, provided that the generators $T$ and $I_{-}$of $D_{6}$ satisfy conditions (i)-(iii) of Theorem 3.3.

Note that when $\Gamma$ is a generic torus, $T$ is necessarily a translation and $I_{-} T$ is necessarily an involution. Thus critically resolvable implies symmetric in this case.
Remark. The prototype symmetric correspondence with graph an elliptic curve (torus) is that which appears in Poncelet's Porism [Berger 1987]. Given any pair of (nonintersecting) real conics $C_{1}$ and $C_{2}$, consider the iteration defined by sending a ray from a point of $C_{1}$ along a direction tangent to $C_{2}$, then repeating the process at the point where the ray (again) hits $C_{1}$. Poncelet's Porism states that whether the ray eventually returns to the initial point depends only on $C_{1}$ and $C_{2}$, and not on the initial point chosen. If one considers the set of pairs of points on $C_{1}$ that lie at opposite ends of tangents to $C_{2}$, one finds that these pairs define (after complexification) an elliptic curve, the graph of the symmetric correspondence defined by sending each point on $C_{1}$ to the opposite ends of the two tangents to $C_{2}$ through the point. Since on the graph the lift $T$ of the correspondence is a translation, we deduce Poncelet's Porism. If $T$ has order three (that is, if $C_{2}$ is triangularly circumscribed in $C_{1}$ ), the correspondence described above is cyclically symmetric.

The description in terms of group actions provides a method of writing down explicit forms for the graph correspondences $\tilde{f}$ that are lifts of critically resolvable correspondences $f$. It is also useful to have canonical forms for $f$ itself. In particular we shall need these later for cyclically symmetric correspondences.

Lemma 3.6. Every cyclically symmetric quadratic correspondence is conformally conjugate to one of the following:

(a) $(w-j z)\left(w-j^{2} z\right)=0$, where $j=e^{2 \pi i / 3}$;

(b) $w^{2}+z w+z^{2}=3$; or

(c) $w^{2}(z+1)+w\left(z^{2}+(a+1) z+a\right)+\left(z^{2}+a z\right)=0$ for some complex value of $a \neq 0,1,9$.

Proof. This follows from normalising the rational cubic $C$ to the three standard forms below, for the three types of graph, by placing the branch points (critical values of the correspondence) at particular locations:

(a) graph two spheres: $C(z)=z^{3}$; 
(b) graph one sphere: $C(z)=z^{3}-3 z$;

(c) graph a torus: $C(z)=z^{2}(z+a) /(z+1)$ for $a \neq 0,1,9$.

Remark. By Lemma 2.2, any map of triples can be decomposed into a cyclically symmetric correspondence followed by a Möbius transformation. Thus Lemma 3.6 yields canonical forms for maps of triples and for reversible maps of triples. We shall make use of these canonical forms in Section 6 .

\section{LIMIT SETS, REGULAR SETS, DIRECTIONALITIES AND FUNDAMENTAL DOMAINS}

The resolvable correspondences dealt with in Section 3 have all grand orbits either finite or dense on $\hat{\mathbb{C}}$. In the present section we consider more general correspondences, and the question of the existence of regular sets and limit sets.

The notions of Julia set for rational maps and of limit set for Kleinian groups do not have a single all-embracing generalisation for correspondences. Rather, we have a chain of limit sets

$$
L_{0}(f) \subseteq J_{0}(f) \subseteq \Lambda_{0}(f) \subseteq \Lambda(f)
$$

defined in different ways, but where certain of the inclusions in the chain become equalities in favourable circumstances. Full details will be presented in [Bullett and Penrose a], but we summarise our present state of knowledge here.

We need two notions to define the various sets in the chain. The omega limit set of $z \in X$, where $X$ is the space on which the correspondence $f$ acts, is

$$
\omega(z)=\overline{\overline{f^{*}(z)}-f^{*}(z)}
$$

where $f^{*}(z)$ denotes the grand orbit of $z$ under $f$ on $X$. The accumulator set of $z \in X$ is

$$
\omega^{-1}(z)=\{w \in X: z \in \omega(w)\}
$$

The minimal limit set of $f$ is

$$
L_{0}(f)=\left\{z: \varnothing \neq \omega^{-1}(z)=X-E(f)\right\},
$$

where $E(f)$ is the exceptional set of points $z \in X$ having finite grand orbit. We conjecture that $L_{0}(f)$ is the intersection of all infinite closed completely invariant subsets of $X$, and that $J_{0}(f) \neq \varnothing$ if and only if $L_{0}(f) \neq \varnothing$.

The generic limit set is

$$
J_{0}(f)=\left\{z:{\overline{\omega^{-1}(z)}}^{\circ} \neq \varnothing\right\}
$$

For a hyperbolic rational map this is the conventional Julia set together with the grand orbits of attracting or superattracting cycles. We conjecture that $J_{0}(f)$ is always closed.

The global accumulation set is

$$
\Lambda_{0}(f)=\left\{z: \omega^{-1}(z) \neq \varnothing\right\}
$$

This set need not be closed. For example, the grand orbit of the centre of a Siegel disc does not lie in $\Lambda_{0}(f)$, whereas the grand orbit of every other point on the disc does.

The domain of proper discontinuity or regular set $\Omega(f)$ is the set of points $z \in X$ having a neighbourhood $U$ with only a finite number of distinct returns under (mixed) iterates of $f$ and $f^{-1}$. Quotienting $\Omega(f)$ by grand orbit equivalence gives a Hausdorff space.

The global limit set $\Lambda(f)$ is the complement of $\Omega(f)$. We conjecture that $\Lambda(f)$ is always the closure of $\Lambda_{0}(f)$.

In analogy with the classical theory for rational maps, it also seems reasonable to define the shadowing-equicontinuity or normality set $N(f)$ as the set of $z \in X$ for which given $\varepsilon>0$ there exists $\delta>0$ such that when $z$ moves a distance less than $\delta$ any point on the grand orbit $f^{*} z$ moves less than $\varepsilon$ (see [Bullett and Penrose a] for more details). The complement of $N(f)$ has some right to the title of Julia set, and we denote it $J(f)$. In [Bullett and Penrose a] it is shown that $J(f) \subset \Lambda(f)$. If no grand orbit of $f$ is dense in an open set of $X$, then $J_{0}(f) \subseteq J(f)$ (possibly with equality in all cases), but the existence of grand orbits dense on open sets completely changes the picture, and there may then be a large part of $J_{0}(f)$ contained in $N(f)$; see Example 4.3 below. In [Bullett and Penrose a] we 
present more details, but all terminology remains provisional until the situation is further clarified.

Example 4.1. If the orbits of $f$ are those of a Kleinian group $G$, then $L_{0}(f)=\Lambda(f)=\Lambda(G)$, the limit set of $G$ in the Kleinian group sense. Also the shadowing-equicontinuity set $N(f)$ is just the regular set $\Omega(G)$ in the Kleinian group sense.

Example 4.2. If $f$ is a rational map, $L_{0}(f)$ is the Julia set of $f$ in the usual rational map sense, $J_{0}(f)$ adds to $L_{0}(f)$ the grand orbits of attracting and superattracting periodic cycles, $\Lambda_{0}(f)$ further adds the basins of superattracting cycles and the grand orbits of cycles of Siegel discs, except for the grand orbits of centres of Siegel discs, and $\Lambda(f)$ finally adds these grand orbits of centres. The shadowingequicontinuity set $N(f)$ is the usual Fatou set for a rational map, minus grand orbits of attracting or superattracting cycles. In other words, $J(f)=$ $J_{0}(f)$.

Example 4.3. If $f$ is the arithmetic-geometric mean correspondence $(2.4)$, then $L_{0}(f)$ is the whole of the Riemann sphere $\hat{\mathbb{C}}$ and thus so are $J_{0}(f), \Lambda_{0}(f)$ and $\Lambda(f)$. At the same time, the shadowing-equicontinuity set $N(f)$ is all of $\hat{\mathbb{C}}$ except for the critical orbit $\{1,-1,0, \infty\}$, as can easily be verified by lifting $f$ to $\tau \mapsto 2 \tau$ on the universal cover of the four-punctured sphere.

Remark. The shadowing-equicontinuity set appears to be the best set on which to generalise Ahlfors' finiteness theorem and Sullivan's analogues for rational maps [Sullivan 1984; 1985a].

We now move on to the more practical problem of identifying regions on which a correspondence acts discontinuously. The dynamics of relations $f$ on general Hausdorff spaces $X$ have been considered by McGehee [1992]. We adapt his approach to our specific situation of algebraic correspondences, and find that as well as enabling us to identify "fundamental domains" for correspondence actions on certain regions, it also enables us to show that certain correspondences have "polynomial-like" actions on other regions, and to deduce, using the theory of [Douady and Hubbard 1985], that the limit sets of these correspondences are made up of copies of Julia sets of polynomial maps.

We make some changes from McGehee's notation: in particular we write $f^{-1}(S)$ rather than $f^{*}(S)$ for the set of points with at least one image under $f$ in $S$, and we reserve the notation $f^{*}(S)$ for the union of grand orbits of points in $S$.

We say that a subset $S \subset X$ defines a directionality for $f$ if $f(\bar{S}) \subset \stackrel{\circ}{S}$. An equivalent way to express this condition is to require that

$$
\left(\bar{S} \times \overline{S^{c}}\right) \cap f=\varnothing
$$

so the condition is also equivalent to $f^{-1}\left(\overline{S^{c}}\right) \subset$ $\left(S^{c}\right)^{\circ}$. The existence of a directionality ensures the existence of an attractor $\Lambda_{+}(f, S)=\bigcap_{n>0} f^{n}(S)$ and a repeller $\Lambda_{-}(f, S)=\bigcap_{n>0} f^{-n}\left(S^{c}\right)$. The attractor and repeller will in general depend on our choice of $S$, but we conjecture them to be unique when $f$ is $(2,2), X$ is the Riemann sphere and $S$ a Jordan disc [Bullett and Penrose a].

Forward orbits started in $S-f(S)$ never return to it, but accumulate on some subset of $\Lambda_{+}(f, S)$. Similarly backward orbits started in $S^{c}-f^{-1}\left(S^{c}\right)$ accumulate on some subset of $\Lambda_{-}(f, S)$. In this sense $S-f(S)$ and $S^{c}-f^{-1}\left(S^{c}\right)$ behave rather like fundamental domains for the respective actions of $f$ and $f^{-1}$ on appropriate regions of X. However what we are really concerned with is arbitrarily mixed iteration under $f$ and $f^{-1}$.

Behaviour under mixed iteration depends on the diagram conditions (if any) satisfied by $f$, and we confine ourselves here to a discussion of two cases that particularly interest us - maps of pairs and reversible maps of triples. In each case we ask that the directionality $S$ satisfy extra hypotheses related to the diagram condition, we define a global attractor $\Lambda(f, S)$ associated to $S$, and we seek a fundamental domain $\Delta$ for the action of $f$ on the complement $\Omega(f, S)$ of $\Lambda(f, S)$ in the usual sense: the union of all images of $\Delta$ under the full action of the correspondence should fill $\Omega(f, S)$, with no two images meeting except at common boundaries. 
The existence of such a $\Delta$ will ensure that $\Omega(f, S)$ is contained in the regular set $\Omega(f)$ and thus that the global limit set $\Lambda(f)$ is contained in $\Lambda(f, S)$. In general in these circumstances $\Lambda(f, S)$ is a filled-in version of $\Lambda(f)$, and in the particular situations of Theorems 5.1 and 6.2 below (and also conjecturally in that of Theorem 6.1), its boundary $\partial \Lambda(f, S)$ is the minimal limit set $L_{0}(f)$.

When $f$ is a map of pairs, we define the global attractor $\Lambda(f, S)$ as follows. We construct a new correspondence $\mathcal{F}$ on the disjoint union $X^{+} \cup X^{-}$ of two copies of the dynamical space $X$ of $f$, with the property that the directed orbits of $\mathcal{F}$ through (copies $z^{+}$or $z^{-}$of) a point $z \in X$ account precisely for all (copies of) points on the grand orbit of $z$ under $f$ (the two copies of $X$ are needed to keep track of switches between "forward mode" and "backward mode"). A bi-injective directionality for $f$ (defined below) gives rise to a directionality $\Sigma$ for $\mathcal{F}$ such that

$$
\pi\left(\Lambda_{+}(\mathcal{F}, \Sigma)\right)=\pi\left(\Lambda_{-}(\mathcal{F}, \Sigma)\right)
$$

where $\pi$ is the projection from $X^{+} \cup X^{-}$to $X$, and we define $\Lambda(f, S)$ to be this set. See [Bullett and Penrose a] for details on all of this.

When $f$ is a reversible map of triples, the definition of $\Lambda(f, S)$ is more straightforward. We ask that $S$ be an equivariant directionality (see the definition below) and it then follows [Bullett and Penrose a] that $\Lambda_{+}(f, S) \cup \Lambda_{-}(f, S)$ is completely invariant under $f$. We define $\Lambda(f, S)$ as this union.

Remark. The construction of $\mathcal{F}$ for a map of pairs $f$ (and similar constructions for other diagram conditions) amounts to organising an ordered exploration of a generic grand orbit of $f$, visiting each point on the orbit exactly once. Finding a fundamental domain for directed iteration of $\mathcal{F}$ amounts to doing this in a way that is continuous almost everywhere. In the case of a group of Möbius transformations, the possibility of performing such a continuous (almost everywhere) ordered exploration is equivalent to discreteness of the group [Sullivan 1982].

\section{Bi-injective directionalities for maps of pairs}

By Lemma 2.1, any map of pairs on the Riemann sphere is a composition $g=Q_{-}^{-1} Q_{+}$, where $Q_{-}$ and $Q_{+}$are rational functions of degree two, and hence $g=\tilde{f}$, the lift of the $(2,2)$ correspondence $f=Q_{+} Q_{-}^{-1}$ to the normalisation $\Gamma$ of its graph.

A bi-injective directionality for the map of pairs $\tilde{f}$ is a pair of subsets $D_{1}, D_{2} \subset \Gamma$ such that:

(i) $Q_{+}\left(D_{1}\right)$ and $Q_{-}\left(D_{2}\right)$ partition the (downstairs) sphere;

(ii) the interiors of $D_{1}$ and $D_{2}$ together cover $\Gamma$; and (iii) $Q_{+}$is injective on $D_{1}$ and $Q_{-}$is injective on $D_{2}$.

Note that (i) and (ii) imply that $D_{1}$ defines a directionality for $\tilde{f}$ in the previous sense. Condition (iii) ensures, among other things, that $\stackrel{\circ}{D}_{1}$ and $\stackrel{\circ}{D}_{2}$ are contained in fundamental domains for $I_{+}$and $I_{-}$, the covering involutions for $Q_{+}$and $Q_{-}$, and (ii) now tells us, by Klein's Combination Theorem [Maskit 1987], that $I_{+}$and $I_{-}$generate a faithful action of the free product $C_{2} * C_{2}$, with the composition $I_{-} I_{+}$a loxodromic element.

Figure 1 illustrates the graph of a real piecewiselinear $(2,2)$ correspondence $f=Q_{+} Q_{-}^{-1}$, and a biinjective directionality for the lift

$$
\tilde{f}=Q_{-}^{-1} Q_{+}
$$

of $f$ to the normalisation $\Gamma$ of this graph (the figure of eight with its crossing point resolved). Note that in the figure the maps $Q_{+}$and $Q_{-}$are projections of the graph of $f$ onto the vertical and horizontal axes respectively.

Theorem 4.4 [Bullett and Penrose a]. If the map of pairs $\tilde{f}$ has a bi-injective directionality defined by $D_{1}$ and $D_{2}$, the intersection $\stackrel{\circ}{D}_{1} \cap \stackrel{\circ}{D}_{2}$ is a fundamental domain for the full action (forwards, backwards and mixed) of $\tilde{f}$ on $\Omega\left(\tilde{f}, D_{1}\right)$, the complement of the global attractor $\Lambda\left(\tilde{f}, D_{1}\right)$.

In particular, $\Omega\left(\tilde{f}, D_{1}\right)$ is contained in the regular set $\Omega(\tilde{f})$. 


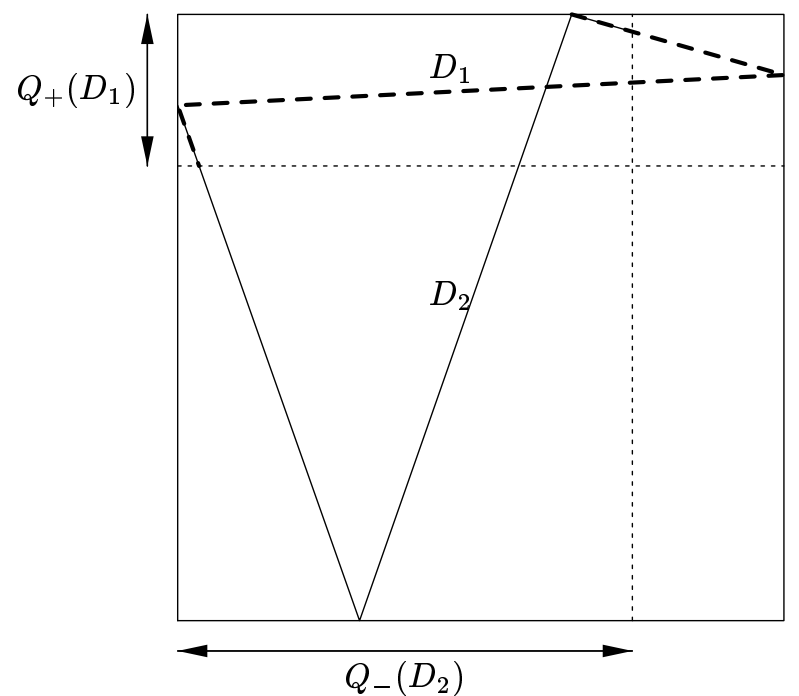

FIGURE 1. A bi-injective directionality for a real piecewise linear map of pairs $\tilde{f}=Q_{-}^{-1} Q_{+}$. Note how the ends of $D_{1}$ (thick dashed line) and $D_{2}$ (thin solid line) overlap.

\section{Equivariant Directionalities for Reversible Maps of Triples}

A reversible map of triples $f$ can be written as a cyclically symmetric correspondence followed by an involution $J$ on $\hat{\mathbb{C}}$, by Lemma 2.2 and the definition of cyclically symmetric in Section 3. For such a correspondence, the involutions $I_{-}$and $I_{+}$ generate a dihedral group $D_{6}$ of order 6 , by the remark following diagram (2.6). (Note that $I_{+}$and $I_{-}$act on the graph $f$; in the case of maps of pairs we were considering this as the dynamical space of $\tilde{f}$.) We say that a subset $D$ of the Riemann sphere defines an equivariant directionality [Bullett and Penrose 1994] for $f$ if:

(i) $D$ is the projection $Q_{-}\left(\overline{\Delta_{0}}\right)$ of the closure of a fundamental domain $\Delta_{0}$ for the action of the $D_{6}$ generated by $I_{-}, I_{+}$on $\Gamma$; and

(ii) $f(\bar{D}) \subset \stackrel{\circ}{D}$ (thus $D$ defines a directionality in the ordinary sense, i.e., $\left(\bar{D} \times \overline{D^{c}}\right) \cap f$ is empty).

We say that $f$ defines an equivariant contact directionality if the second condition is modified to

(ii) $^{\prime}\left(\bar{D} \times \overline{D^{c}}\right) \cap f=\left\{\left(z_{0}, z_{0}\right)\right\}$ for a single point $z_{0}$.

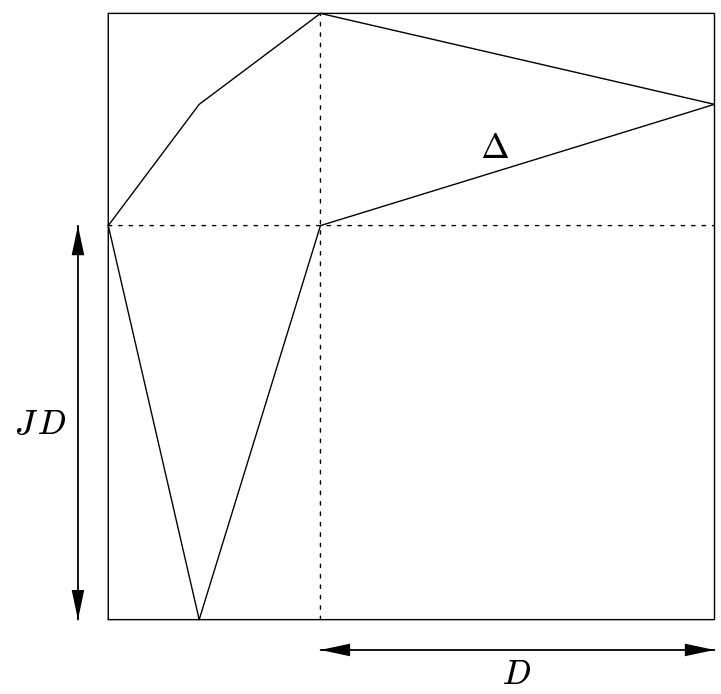

FIGURE 2. Equivariant directionality for a reversible real piecewise linear map of triples $f=Q_{+} Q_{-}^{-1}$.

This new condition allows $f(D)$ to have boundary meeting that of $D$, but only at a single point, and moreover requires that this single point be a fixed point of $f$. The "attractor" and "repeller" will also meet at this special contact point.

Figure 2 provides an illustration of the graph $f$ of a real piecewise linear $(2,2)$ correspondence with an equivariant directionality.

Theorem 4.5 [Bullett and Penrose a]. Suppose D defines an equivariant directionality or contact directionality for the reversible map of triples $f$. Then $\Lambda_{-}(f, D)$ is the image of $\Lambda_{+}(f, D)$ under the timereversal symmetry $J$, and the union $\Lambda(f, D)$ of these two sets is fully invariant under $f$. Moreover $f$ acts discontinuously on the complement $\Omega(f, D)$ of $\Lambda(f, D)$, and any fundamental domain $\Delta$ for the action of $J$ on $D \cap J D$ is a fundamental domain for the full action of $f$ on $\Omega(f, D)$.

We now have all the tools we need to investigate regular and limit sets for some specific classes of examples of correspondences. This we do in the remaining sections. 


\section{COMBINING QUADRATIC-LIKE JULIA SETS}

A bi-injective Jordan directionality for a map of pairs $\tilde{f}$ on the Riemann sphere is a bi-injective directionality with the property that $Q_{+}\left(D_{1}\right)$ is a Jordan disc (and hence so is $Q_{-}\left(D_{2}\right)$ ).

Theorem 5.1 [Bullett and Penrose a]. If $\left(D_{1}, D_{2}\right)$ is a bi-injective Jordan directionality for the map of pairs $\tilde{f}$, then:

(i) $\Lambda_{+}\left(\tilde{f}, D_{1}\right)$ and $\Lambda_{-}\left(\tilde{f}, D_{1}\right)$ are hybrid equivalent to filled-in Julia sets $K_{c_{1}}$ and $K_{c_{2}}$ of the family $q_{c}: z \mapsto z^{2}+c$ (possibly Cantor sets).

(ii) On $\Lambda_{-}\left(\tilde{f}, D_{1}\right)$ the correspondence $\tilde{f}$ has a branch conjugate to $q_{c_{2}}$, and on $\Lambda_{+}\left(\tilde{f}, D_{1}\right)$ its inverse $(\tilde{f})^{-1}$ has a branch conjugate to $q_{c_{1}}$.

Recall that a hybrid equivalence [Douady and Hubbard 1985] between $q_{c}$ and a holomorphic map $g$ is a quasiconformal equivalence $\varphi$ such that $\bar{\partial} \varphi=0$ almost everywhere on $K_{c}$.

The proof of this theorem is an application of the theory of polynomial-like mappings [Douady and Hubbard 1985]. It is also shown in [Bullett and Penrose a] that the boundaries of $\Lambda_{+}\left(\tilde{f}, D_{1}\right)$ and $\Lambda_{-}\left(\tilde{f}, D_{1}\right)$ (which by the theorem are copies of quadratic Julia sets $J_{c_{1}}$ and $J_{c_{2}}$ ) can be characterised by the properties that $\partial \Lambda_{+}\left(\tilde{f}, D_{1}\right)$ is the set of all points $z$ having arbitrarily small neighbourhoods $U$ such that $\bigcup_{n>0} \tilde{f}^{-n}(U)$ covers the whole sphere

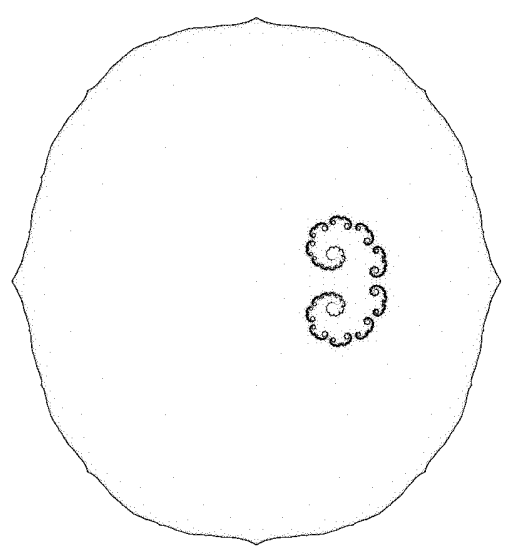

FIGURE 3. Left: forward and backward orbits for (5.1) with parameters $a=0.8, b=-0.15, c=0, d=0.5$. Right: a grand orbit for the same correspondence. except for at most two points, and that $\partial \Lambda_{-}\left(\tilde{f}, D_{1}\right)$ satisfies the analogous condition with $\tilde{f}^{-1}$ in place of $\tilde{f}$.

Any pair of quadratic Julia sets $J_{c_{1}}$ and $J_{c_{2}}$ can be realised as $\partial \Lambda_{+}\left(\tilde{f}, D_{1}\right)$ and $\partial \Lambda_{-}\left(\tilde{f}, D_{1}\right)$ for some map of pairs $\tilde{f}$ with a bi-injective Jordan directionality [Bullett and Penrose a]. Figure 3 (left) displays the set of images of a single point under forward iteration and under backward iteration (but not mixed iteration) for an example in the family

$$
\frac{z(z+a)}{c z(z+a)+1}=\frac{w^{2}}{w+b / a}+a b d
$$

Here $\partial \Lambda_{+}\left(\tilde{f}, D_{1}\right)$ and $\partial \Lambda_{-}\left(\tilde{f}, D_{1}\right)$ are the omega limit sets of the forward and backward orbits respectively. In Figure 3 (right) we display a single grand orbit, the omega limit set of which is $\partial \Lambda\left(\tilde{f}, D_{1}\right)$, for the same correspondence.

Observe that in this case the global attractor $\Lambda\left(\tilde{f}, D_{1}\right)$ is the closure of a disjoint union of copies of $\Lambda_{+}\left(\tilde{f}, D_{1}\right)$ and $\Lambda_{-}\left(\tilde{f}, D_{1}\right)$. We show in [Bullett and Penrose a] that this is true in general in the situation of Theorem 5.1, provided that $\Lambda_{+}$ and $\Lambda_{-}$are connected, and true in certain cases (such as that illustrated in Figure 3) when one or both of these sets is not connected. Moreover in all these cases $\partial \Lambda\left(\tilde{f}, D_{1}\right)$ is the minimal limit set $L_{0}(\tilde{f})$.

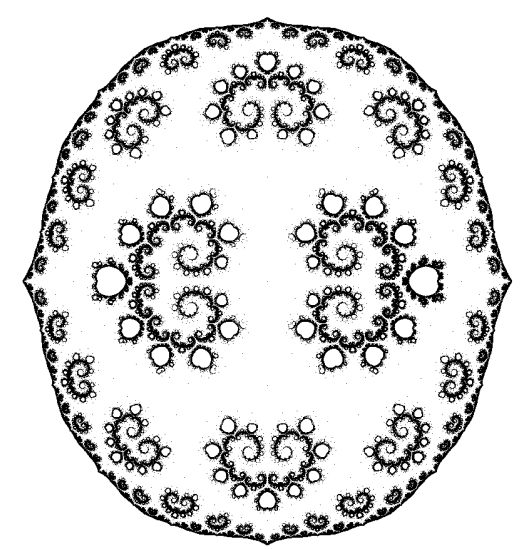



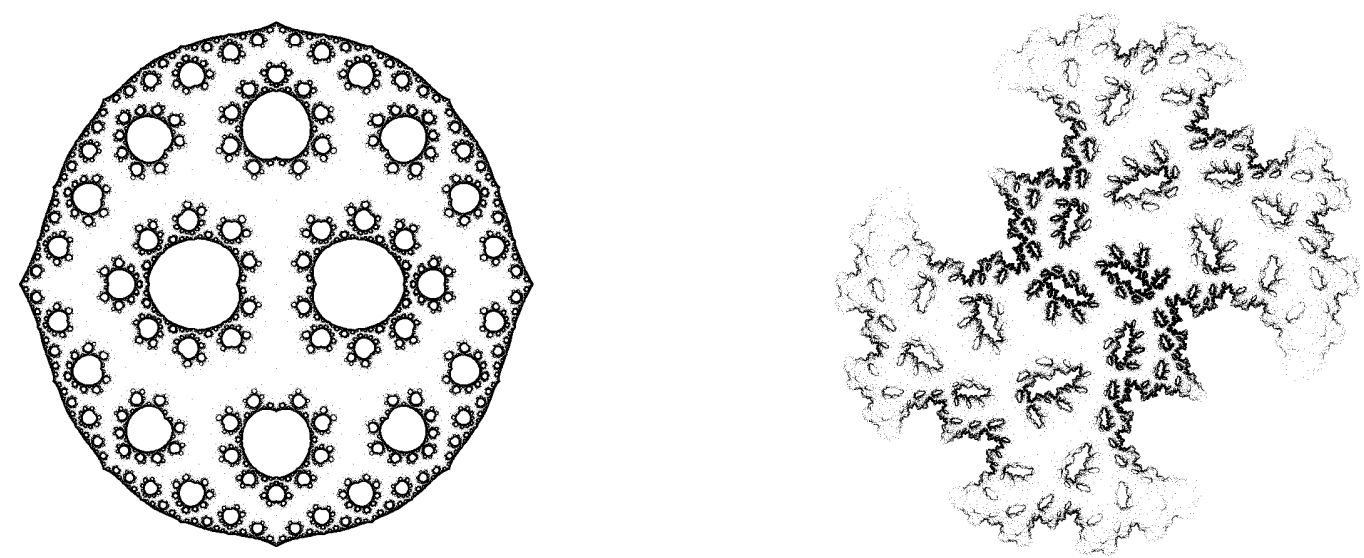

FIGURE 4. Left: a grand orbit for (5.1) with parameters $a=0.8, b=-0.15, c=0, d=0$. Right: a grand orbit for $a=0.8, b=-0.15, c=-0.35-0.55 i, d=-0.35+0.55 i$. Both examples are reversible, and in particular the forward and backward limit sets are isomorphic. For the picture on the right each is a copy of Douady's rabbit.

Figure 4 illustrates $\partial \Lambda\left(\tilde{f}, D_{1}\right)=L_{0}(\tilde{f})$ for two other examples, in each of which $\Lambda_{+}$and $\Lambda_{-}$are connected and isomorphic.

\section{MATING QUADRATIC MAPS WITH THE MODULAR GROUP}

By Lemma 3.6 and the subsequent remark, a reversible map of triples with graph a pair of spheres is equivalent to one that can be written

$$
(J z)^{2}+(J z) w+w^{2}=0,
$$

where $J$ is an involution. Thus it factorises into a pair of Möbius transformations

$$
w=e^{ \pm 2 \pi i / 3} J z .
$$

It follows that there is a bijection between equivalence classes of such correspondences and conjugacy classes of representations of the modular group $\operatorname{PSL}(2, \mathbb{Z})$ in $\operatorname{PSL}(2, \mathbb{C})$, since $\operatorname{PSL}(2, \mathbb{Z})$ is the free product of a cyclic group of order two and one of order three, being generated by the matrices $\left(\begin{array}{rr}0 & 1 \\ -1 & 0\end{array}\right)$ and $\left(\begin{array}{rr}0 & -1 \\ 1 & 1\end{array}\right)$.

Again by the same lemma and remark, a reversible map of triples with graph a single sphere is equivalent to one that can be written

$$
(J z)^{2}+(J z) w+w^{2}=3,
$$

with $J$ an involution. One parametrisation for these equivalence classes is to represent them as $f_{a, k}: z \mapsto w$, where [Bullett and Penrose 1994]

$$
\left(\frac{a z+1}{z+1}\right)^{2}+\left(\frac{a z+1}{z+1}\right)\left(\frac{a w-1}{w-1}\right)+\left(\frac{a w-1}{w-1}\right)^{2}=3 k,
$$

with parameters $a, k \in \mathbb{C}$. This exhibits the timereversal symmetry in the form $z \mapsto-z$.

We now specialise to the one (complex) parameter family $f_{a}$ defined by (6.1) when $k=1$. This is the so-called contact condition [Bullett and Penrose 1994], namely that the fixed point 0 of the time-reversal symmetry be also a fixed point for the correspondence. In [Bullett and Penrose 1994] we show that for real $a$ with $4 \leq a \leq 7$, and conjecturally for all $a$ in a set $M$ of parameter values resembling the Mandelbrot set, this correspondence is a mating of a quadratic map with the modular group, in the following sense.

Theorem 6.1 [Bullett and Penrose 1994]. For a real, $4 \leq a \leq 7$ and $k=1$, the Riemann sphere is partitioned into two subsets $\Omega$ and $\Lambda$, both fully invariant under $f_{a}$, such that:

(i) $\Omega$ is conformally equivalent to the (open) complex upper half-plane, and the action of $f_{a}$ on it is conjugate to that of the generators $z \mapsto z+1$ 
and $z \mapsto z /(z+1)$ of $\operatorname{PSL}(2, \mathbb{Z})$ on the halfplane;

(ii) $\Lambda$ is the union of closed simply connected subsets $\Lambda_{+}$and $\Lambda_{-}$, which meet at a single point. These sets are forward and backward invariant, respectively. Moreover, $f_{a}$ has a branch mapping $\Lambda_{-}$onto itself with degree two, and $f_{a}^{-1}$ has a branch mapping $\Lambda_{+}$onto itself with degree two. The remaining forward branch of $f_{a}$ on $\Lambda_{+} \cup \Lambda_{-}$sends $\Lambda_{-}$homeomorphically onto $\Lambda_{+}$.

The sets $\Lambda_{+}$and $\Lambda_{-}$appear to be homeomorphic to $K_{c}$ (for some $c$ in the Mandelbrot set), and appropriate branches of $f_{a}$ and $f_{a}^{-1}$ appear to be topologically conjugate to $q_{c}$ on appropriate regions. There are technical difficulties in establishing a formal proof (see [Bullett and Penrose 1994]) and so far we have succeeded in proving this only for small perturbations of $k$ away from $k=1$ (but the correspondence then no longer acts on $\Omega$ as a group):

Theorem 6.2 [Bullett and Penrose 1994]. For $4<$ $a<7$ and sufficiently small perturbations $k=1-$ $\varepsilon$ of $k=1$ (with $\varepsilon>0$ ), the Riemann sphere is partitioned into two subsets $\Omega$ and $\Lambda$, both fully invariant under $f_{a, k}$, such that:

(i) $\Omega$ is conformally equivalent to an annulus, and the action of $f$ on it is discontinuous;

(ii) $\Lambda$ is the disjoint union of $\Lambda_{+}$and $\Lambda_{-}$, which are forward and backward invariant respectively. On a neighbourhood of $\Lambda_{-}$there is a branch of $f$ that is hybrid equivalent to a quadratic map $q_{c}: z \mapsto z^{2}+c$, with a connected filled-in Julia set $K_{c}$; this hybrid equivalence sends $\Lambda_{-}$to $K_{c}$ by a quasiconformal bijection. On a neighbourhood of $\Lambda_{+}$there is a branch of $f^{-1}$ with the same property (for the same $c$ ). The remaining forward branch of $f$ on $\Lambda$ sends $\Lambda_{-}$onto $\Lambda_{+}$by a conformal bijection.

The proof of this result, like that of Theorem 5.1, is based on the theory of polynomial-like mappings [Douady and Hubbard 1985]. We conjecture that $\Lambda_{+}$and $\Lambda_{-}$in Theorem 6.1 are also homeomorphic to filled-in quadratic Julia sets $K_{c}$, and that appropriate branches of $f_{a}$ and $f_{a}^{-1}$ are topologically conjugate to $q_{c}$ on them. It therefore seems reasonable to describe the correspondences $f_{a}$ as topological matings of the modular group with $q_{c}$, matings in which the the generators of $z \mapsto z+1$, $z \mapsto z /(z+1)$ of $\operatorname{PSL}(2, \mathbb{C})$ acting on the boundary of the complex upper half-plane are matched with those of $q_{c}^{-1}: z \mapsto \sqrt{z-c}$ on the Julia set $J_{c}$ bounding $K_{c}$. It appears that every connected Julia set $K_{c}$ in the quadratic family can be realised in this way. Figures 5, 6 and 7 illustrate examples.

Remark. For $a=4$ and $k=1$ the correspondence is critically resolvable (Section 3 ) and therefore its lift to its graph $f$ has orbits those of a group. This group is $\mathrm{PGL}(2, \mathbb{Z})$, with the standard action [Bullett and Penrose 1994]. For other values of $a \in M$ (and $k=1$ ), the lifted correspondence continues to act as $\operatorname{PGL}(2, \mathbb{Z})$ on the lift $\tilde{\Omega}$ of $\Omega$, which remains conformally a pair of open discs. Thus we may regard $M$ as parametrising a family of perturbations of $\operatorname{PGL}(2, \mathbb{Z})$ as a correspondence, the action remaining unchanged on a pair of regular domains.

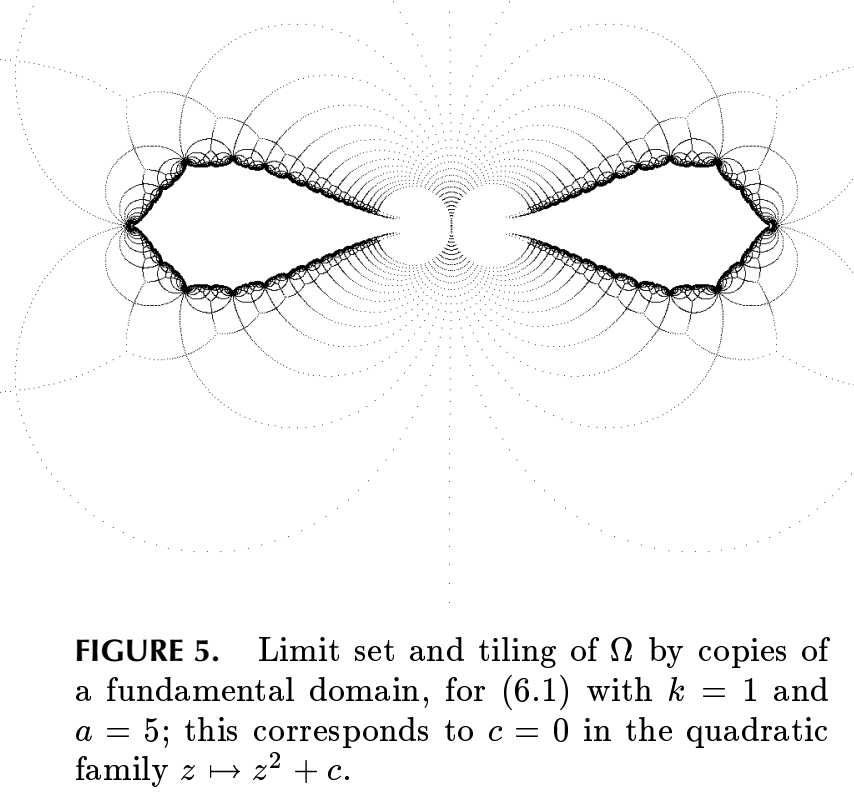




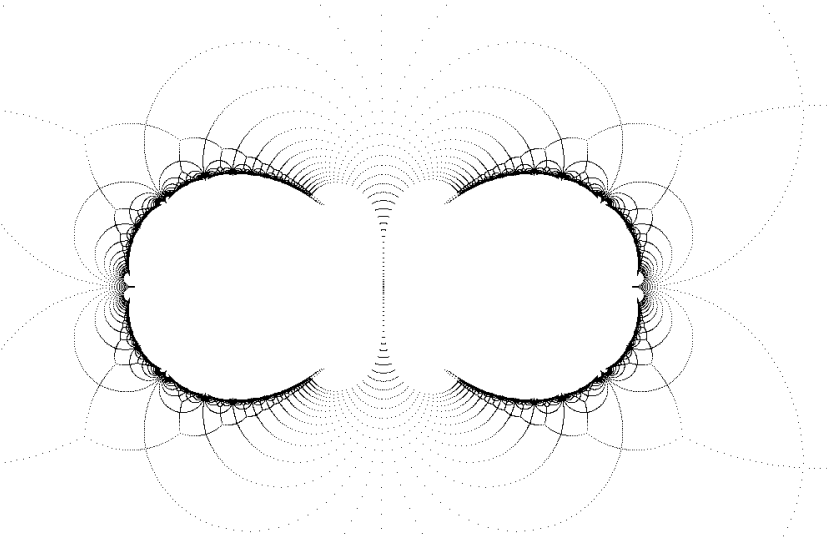

FIGURE 6. Limit set and tiling of $\Omega$ for (6.1) with $k=1$ and $a=7$; this corresponds to $c=1 / 4$ in the quadratic family. The limit set appears to be a quasicircle (unlike the Julia set of the quadratic map: see [Bullett and Penrose 1994] for an explanation). the plot is incomplete near the origin for computational reasons.

FIGURE 7. Limit set and tiling of $\Omega$ for (6.1) with $k=1$ and $a=4.54+0.44 i$; a mating of the modular group with Douady's rabbit.

\section{PERTURBING CIRCLE PACKING KLEINIAN GROUPS AS CORRESPONDENCES}

Consider the two-generator subgroups of $\operatorname{PSL}(2, \mathbb{C})$ generated by Möbius transformations $z \mapsto A z$ and $z \mapsto B z$ such that $B A^{-1}$ is an involution (this is the condition that the correspondence be a map of pairs), and that there exists an involution $N$ such that $B=N A N$ (this is a time-preserving involution of the correspondence). It is shown in [Bullett and Penrose b] that there is a one (complex) parameter moduli space of such groups, in particular that each conjugacy class contains a group generated by a pair of maps $A, B: z \mapsto w$ satisfying a relation

$$
\left(\frac{\tau}{w}-\frac{w}{\tau}\right)\left(z-\frac{1}{z}\right)=4
$$

and that, for each conjugacy class, $\tau \in \mathbb{C}-\{0\}$ is unique up to $\tau \leftrightarrow 1 / \tau$. These groups come equipped with certain extra structure. For example, for every two-generator subgroup of $\operatorname{PSL}(2, \mathbb{C})$ as described (and almost all others) there is an involution $J$ such that $J A J=A^{-1}$ and $J B J=B^{-1}$ [Bullett and Penrose b]. We can define a new timereversing involution $K$ by $K=J N$, and it is then a short exercise in algebra to show that $(K A)^{4}$ is the identity, and that the one-parameter family of groups defined above is simply the moduli space of all representations of the free product $C_{4} * C_{2}$ (generated by $K A$ and $K$ respectively). Note that $\langle A, B\rangle$ has index two in $\langle A, B, K\rangle=\langle K A, K\rangle$, since $B=K(K A)^{-1}$. Explicitly, in the parametrisation above, we have $A=\left(\begin{array}{cc}\tau & -\tau \\ 1 & 1\end{array}\right), B=\left(\begin{array}{rr}\tau & \tau \\ -1 & 1\end{array}\right)$, $N=\left(\begin{array}{rr}-1 & 0 \\ 0 & 1\end{array}\right), J=\left(\begin{array}{ll}0 & \tau \\ 1 & 0\end{array}\right), K=\left(\begin{array}{cc}0 & -\tau \\ 1 & 0\end{array}\right)$, and $K A=$ $\left(\begin{array}{rr}-1 & -1 \\ 1 & -1\end{array}\right)$.

We can generalise these groups to correspondences retaining the properties of separability and reversibility, and having a time-preserving involution that exchanges the two critical points for the forwards map and simultaneously exchanges the two critical points for the backwards map. Thus we retain $J, N$ and $K$ as in the group case, and obtain a two (complex) parameter family 


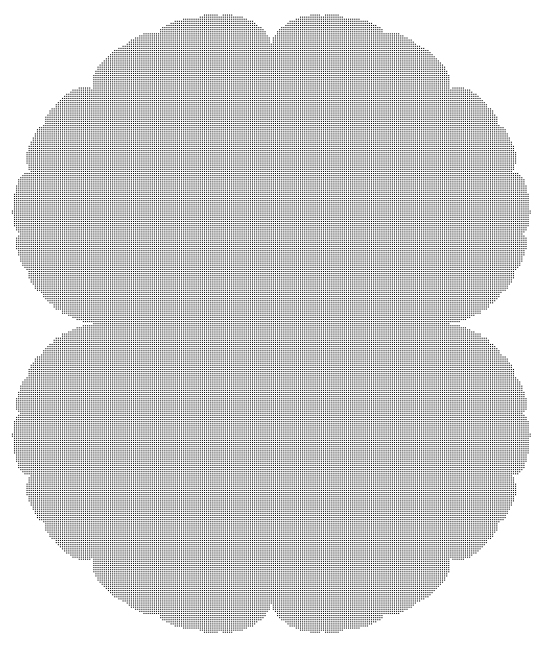

FIGURE 8. The set $\mathcal{T}$ of $\tau$ where the representation (7.1) of $C_{2} * C_{4}$ is faithful and discrete.

$$
\left(\frac{\tau}{w}-\frac{w}{\tau}\right)\left(z-\frac{1}{z}\right)=k .
$$

These are the correspondences among which we shall perturb our representations of $C_{4} * C_{2}$.

The moduli space of representations of $C_{4} * C_{2}$ is already a complicated object. It has a structure analogous to that of the Riley slice [Keen and Series 1991] of representations of $C_{\infty} * C_{\infty}$ with parabolic generators. Let $\mathcal{T}$ denote the subset of parameter space such that for $\tau \in \mathcal{T}$ the group acts discontinuously on an open set $\Omega$ and has a totally disconnected limit set $\Lambda$. It appears (Figure 8) that $\mathcal{T}$ is a once-punctured topological disc, and that on its boundary there is a dense set of values of $\tau$ where the action of $C_{4} * C_{2}$ remains faithful, but where the limit set becomes a circle packing, due to the appropriate group elements becoming parabolic (for related results see [Keen and Series 1992; Keen et al. 1993; McMullen 1991]).

For $\tau$ outside $\mathcal{T}$ the action of $C_{4} * C_{2}$ is no longer discrete, except at some isolated values of $\tau$ where the elements just described become elliptic of finite order. In particular, the representation is no longer faithful. When we regard the action of $C_{4} * C_{2}$ as that of a correspondence $z \mapsto w$, it has a biinjective directionality when $\tau$ is in the interior of
$\mathcal{T}$, but this degenerates into a contact directionality at the boundary points of $\mathcal{T}$ which correspond to circle packings. Here the contact condition is analogous to that of Section 4 when the packing is a single circle (at $\tau=3-2 \sqrt{2}$ ), where it is the condition that one fixed point of $J$ be fixed by the correspondence, but it is a more complicated cycle condition for other circle packings. See [Bullett and Penrose b] for details. Figure 9 illustrates two of the circle packings that occur on the boundary of $\mathcal{T}$.

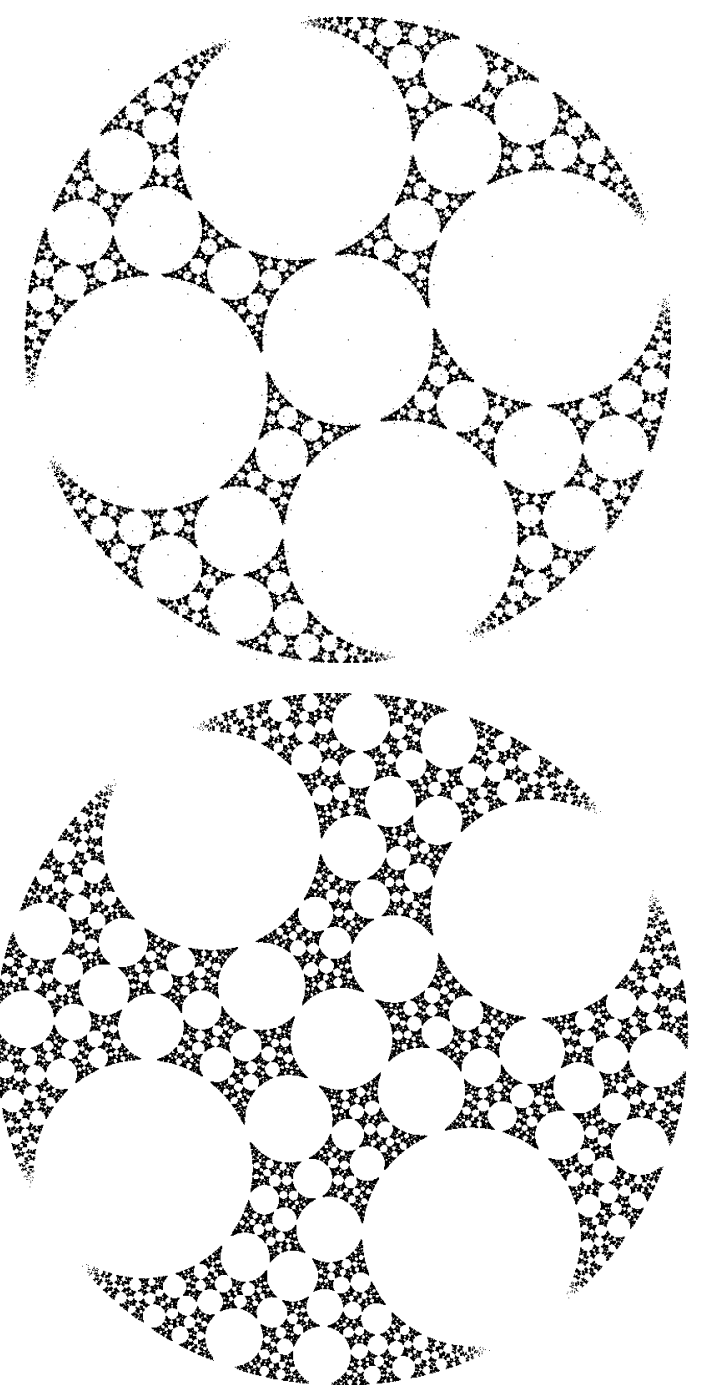

FIGURE 9. Circle packing for correspondences in the family (7.1). Top: $\tau=(2-\sqrt{3}) i$. Bottom: $\tau$ is a solution of $T=\frac{1}{2}\left(\tau+\tau^{-1}+2\right)$, where $T^{3}-4 T^{2}+5 T+2=0$. 
We can perturb $C_{4} * C_{2}$ as a correspondence in such a way as to obtain topologically unchanged limit set behaviour for any $\tau \in \mathcal{T}$, but it is the values of $\tau$ on the boundary of $\mathcal{T}$ that will be of particular interest to us. The reason is that although circle packing actions of Kleinian groups are geometrically rigid [Keen et al. 1993], we are able to deform the limit sets topologically by perturbing the groups as correspondences.

In order to maintain the topological conjugacy type of the limit set we perturb the pair $(k, \tau)$ in such a way as to maintain the contact condition associated to the circle packing in question [Bullett and Penrose b]. In Figure 10 we deform the circle packing group of Figure 9 (top) as a correspondence, maintaining the contact condition that the fixed point $\sqrt{\tau}$ of $J$ map under the correspondence to the fixed point $i \sqrt{\tau}$ of $K$. Algebraically, this condition is $k=\left(\tau^{-1}-\tau\right) i$. On the left, the circles of original packing have been partly "pinched" by pulling an opposite pair of points towards each other, and on the right this pinching process is complete - the circles have now each been divided in two. In principle, any circle packing action of $C_{4} * C_{2}$ can be deformed in this way, though contact conditions become increasingly difficult to compute explicitly for more complicated packings. Similar deformations should be possible for actions of other free products $C_{m} * C_{n}$ of two cyclic groups.

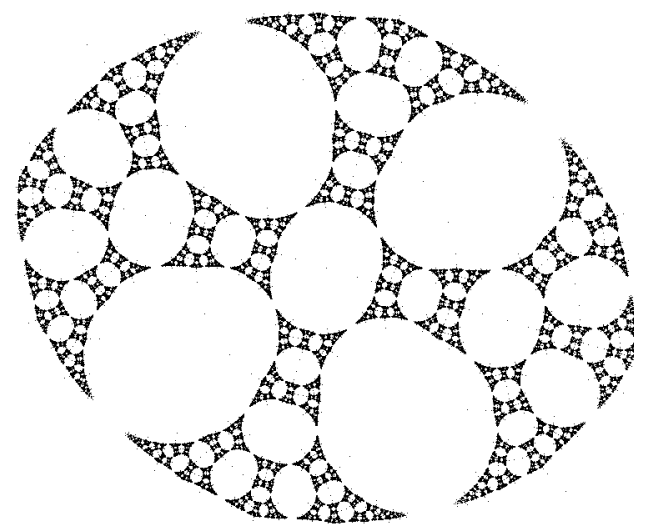

Remark. In all the examples above we have $L_{0}=$ $\Lambda=J$ in the notation of Section 4. In other words, the different definitions of limit set for these correspondences are all equivalent. Note also that when deform away from groups, the correspondences above cease to act as groups on the regular set $\Omega$ (since critical points are introduced there), but continue to act as groups on the limit set $\Lambda$. This should be contrasted with the behaviour we observed for matings of quadratic maps with the modular group, where a group action was retained on the regular set but lost on the limit set.

\section{MORE EXOTIC EXAMPLES: TWEAKED JULIA SETS AND BULL'S-EYES}

The examples in this section have the property that the forward or backward limit set is a "quotient" of a quadratic-like Julia set. We refer to these quotients as tweaked Julia sets. We confine ourselves to a few remarks and otherwise let the pictures speak for themselves.

Suppose our quadratic correspondence $f$ has the property that there is a point $z_{0}$ that is an attractive fixed point for one branch, but for which all other values of $f^{n}\left(z_{0}\right)$, for $n>0$, are bounded away from $z_{0}$. Let $D$ be the immediate basin of attraction of $z_{0}$. The space $S$ of orbits

$$
z_{1} \mapsto z_{2} \mapsto z_{3} \mapsto \cdots
$$

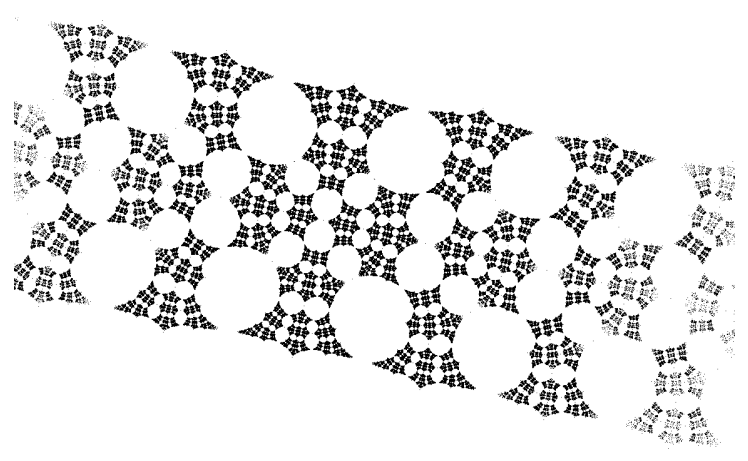

FIGURE 10. Deformations of Figure 9 (top). Left: $k=10 / 3$ and $\tau=i / 3$ in (7.2). Right: $k=(1+\sqrt{17}) / 2$ and $\tau=(k-\sqrt{k}) i / 2$ in $(7.2)$. 

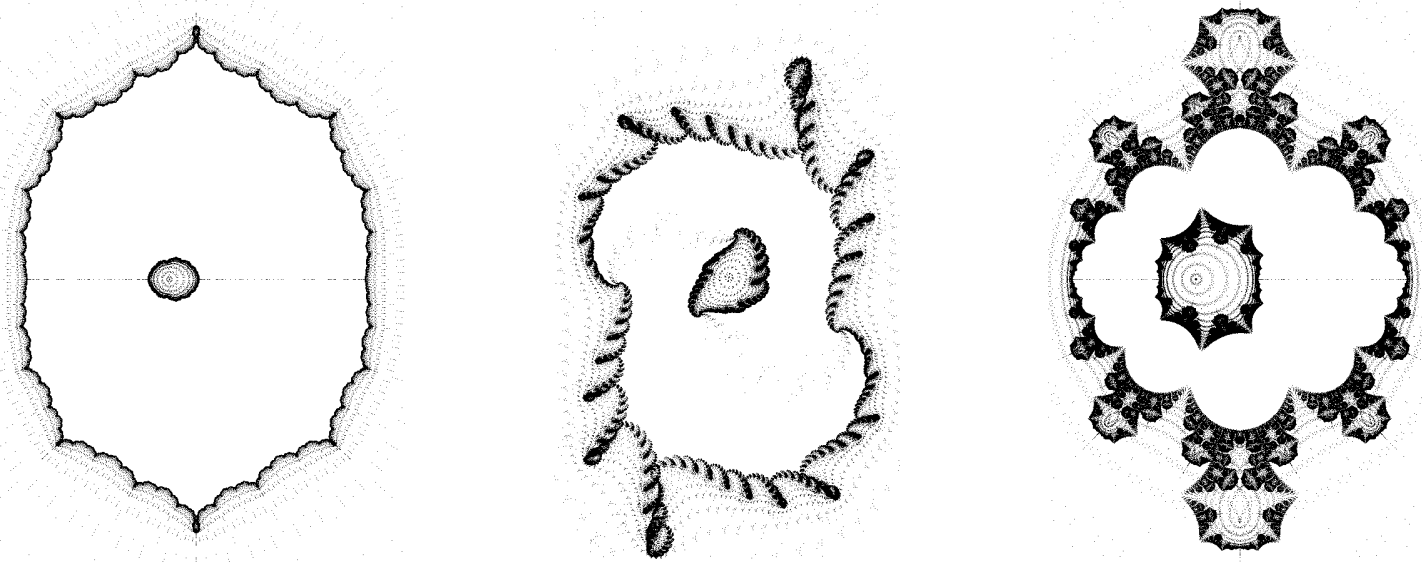

FIGURE 11. Forward images of backward critical point and backward images of the forward critical point, for correspondences in the family (8.1). Left: $a=-4 / 15$ and $b=2 / 5$. Middle: $a=25 i / 78$ and $b=5 / 13-10 i / 39$. Right: $a=-1 / 2$ and $b=1 / 2$.

that converge to $z_{0}$ and have all $z_{i}$ in $D$ is homeomorphic to a disc, but the projection of $S$ onto $D$ (sending each orbit onto its first point $z_{1}$ ) may be many-to-one near the boundary of $D$, as for $z_{1}$ well away from $z_{0}$ there may be several forward routes converging to $z_{0}$. The Julia set that we plot in the dynamical plane by back iteration from a starting point near $z_{0}$ is the projection of the boundary of $S$, so what we see may be a set that appears to cross over itself again and again. This effect can be observed in Figure 11 (middle). The Julia set is a topological circle in orbit space, but is tweaked by projection to the dynamical plane (as is also the case in [Bullett 1988, Fig. 3]).

Similar tweaking can be performed with basins of attraction of periodic points, with increasingly complicated pictures as the period increases. The construction can also produce combinations analogous to those of Section 5, but now in the case that there is only a noninjective directionality. Finally the bull's-eye examples (Figure 11) display the phenomenon of tweaking particularly clearly. These are correspondences in the family

$$
z(z+a)=\frac{w^{2}}{w+b} \quad \text { with } \quad a=\frac{b^{2}}{b-1},
$$

and have the property that the superattractive fixed point $\infty$ has an orbit mapping (in four iterations) to the superrepulsive fixed point 0 . What Figure 11 shows is a complete set of all forward images, up to a certain depth, of the backward critical point $-2 b$, plus all backward images, up to the same depth, of the forward critical point $-a / 2$. The starting point $-2 b$ has a forward orbit taking it close to $\infty$, but at each step when it approaches $\infty$ it has a path of length four leading close to the superrepeller 0 ; points on the path have subsequent images spreading out from near the superrepeller and accumulating on the forward limit set. It is these subsequent images that fall into the bands we see the computer plots. The backward orbits started at $-a / 2$ behave in a similar (time-reversal symmetric) way.

\section{CONCLUDING REMARKS}

What conclusions can be drawn from our gallery of exhibits? First, it should be possible to prove that the examples of Sections 5, 6 and 7 are generically structurally stable, by which we mean that using the techniques developed in [Sullivan 1984; $1985 \mathrm{a} ; 1985 \mathrm{~b}]$ it should be possible to prove that an open dense set of each type of example has a family of perturbations with dynamics that is topologically unchanged, and that these families of perturbations form open sets in the spaces of correspondences satisfying the same constraints as 
the unperturbed examples (i.e., the same diagram conditions).

This phenomenon should provide a bridge between the situation for rational maps, where such stability is now reasonably understood, and that for Kleinian groups, where it is a major unsolved question as to whether structural stability is an open dense property among discrete representations [Sullivan 1985b] (for Kleinian groups, relations play the role of our diagram conditions).

Our matings of the modular group with quadratic maps also offer the possibility of exploiting the bijection between the dynamics of the modular group and that of the shift. This has always been possible at the combinatorial level [Gutzwiller and Mandelbrot 1988], but our examples provide a geometrisation in the realm of complex analysis.

Finally we remark that in this article we have been concerned mainly with discrete actions of correspondences. Another class of correspondences of great potential interest are those exhibiting behaviour of Hamiltonian type. A start on the study of such correspondences was made in [Bullett 1988].

\section{ACKNOWLEDGEMENTS}

We thank both referees for their very helpful suggestions, in particular for proposing general algebraic settings for the study of iterated correspondences.

\section{REFERENCES}

[Beardon 1991] A. F. Beardon, Iteration of Rational Functions, Springer, New York, 1991.

[Berger 1987] M. Berger, Geometry, Springer, Berlin, 1987.

[Bullett 1988] S. Bullett, "Dynamics of quadratic correspondences", Nonlinearity 1 (1988), 27-50.

[Bullett 1991] S. Bullett, "Dynamics of the arithmeticgeometric mean", Topology 30 (1991), 171-190.

[Bullett 1992] S. Bullett, "Critically finite correspondences, and subgroups of the modular group", Proc. Lond. Math. Soc. (3) 65 (1992), 423-448.
[Bullett and Penrose 1994] S. Bullett and C. Penrose, "Mating quadratic maps with the modular group", Inventiones Math. 115 (1994), 483-511.

[Bullett and Penrose a] S. Bullett and C. Penrose, "Limit sets for correspondences", I and II (in preparation).

[Bullett and Penrose b] S. Bullett and C. Penrose, "Perturbing circle-packing Kleinian groups as correspondences" (in preparation).

[Douady and Hubbard 1982] A. Douady and J. H. Hubbard, "Itérations des polynômes quadratiques complexes", Comptes R. Acad. Sci. Paris 294 (1982), 123-126.

[Douady and Hubbard 1984] A. Douady and J. H. Hubbard, "Étude dynamique des polynômes complexes", Publ. Math. Orsay, I (1984), II (1985).

[Douady and Hubbard 1985] A. Douady and J. H. Hubbard, "On the dynamics of polynomial-like mappings", Ann. Sci. Éc. Norm. Sup. (Paris) $\mathbf{1 8}$ (1985), 287-343.

[Gutzwiller and Mandelbrot 1988] M. C. Gutzwiller and B. B. Mandelbrot, "Invariant multifractal measures in chaotic Hamiltonian systems, and related structures", Phys. Rev. Lett. 60 (1988), 673-676.

[Keen and Series 1991] L. Keen and C. Series, "The Riley slice of Schottky space" (preprint), University of Warwick, 1991.

[Keen and Series 1992] L. Keen and C. Series, "Pleating coordinates for the Teichmüller space of a punctured torus", Bull. Am. Math. Soc. 26 (1992), 141-146.

[Keen et al. 1993] L. Keen, B. Maskit and C. Series, "Geometric finiteness and uniqueness for Kleinian groups with circle packing limit sets", J. reine angew. Math. 436 (1993), 209-219.

[Maskit 1987] B. Maskit, Kleinian groups, Springer, New York, 1987.

[McGehee 1992] R. McGehee, "Attractors for closed relations on compact Hausdorff spaces", Indiana Univ. Math. J. 41 (1992), 1165-1209.

[McMullen 1991] C. T. McMullen, "Cusps are dense", Ann. of Math. 133 (1991), 217-247. 
[Münzner and Rasch 1991] H. F. Münzner and H.-M. Rasch, "Iterated algebraic functions and functional equations", Int. J. Bifurcation and Chaos 1 (1991), 803-822.

[Rasch 1988] H.-M. Rasch, "Über die Iteration algebraischer Funktionen", Dissertation, Univ. Bremen, 1988.

[Semple and Roth 1949] J. G. Semple and L. Roth, Introduction to Algebraic Geometry, Oxford University Press, Oxford, 1949.

[Shafarevich 1974] I. R. Shafarevich, Basic Algebraic Geometry, Springer, Berlin, 1974.
[Sullivan 1984] D. Sullivan, Quasiconformal homeomorphisms and dynamics III (preprint), IHES, 1984.

[Sullivan 1985a] D. Sullivan, "Quasiconformal homeomorphisms and dynamics, I: Solution of the FatouJulia problem on wandering domains", Ann. Math. 122 (1985), 401-418.

[Sullivan 1985b] D. Sullivan, "Quasiconformal homeomorphisms and dynamics, II", Acta Math. 155 (1985), 243-260.

[Sullivan 1982] D. Sullivan, "Discrete conformal groups and measurable dynamics", Bull. Am. Math. Soc. 6 (1982), 57-73.

Shaun Bullett, School of Mathematical Sciences, Queen Mary and Westfield College, University of London, Mile End Road, London E1 4NS, United Kingdom (S.R.Bullett@qmw.ac.uk)

Christopher Penrose, School of Mathematical Sciences, Queen Mary and Westfield College, University of London, Mile End Road, London E1 4NS, United Kingdom

Current address: Department of Mathematical Sciences, Loughborough University of Technology, Loughborough, Leics LE11 3TU, United Kingdom (C.S.Penrose@lut.ac.uk)

Received October 26, 1993; accepted in revised form August 2, 1994 